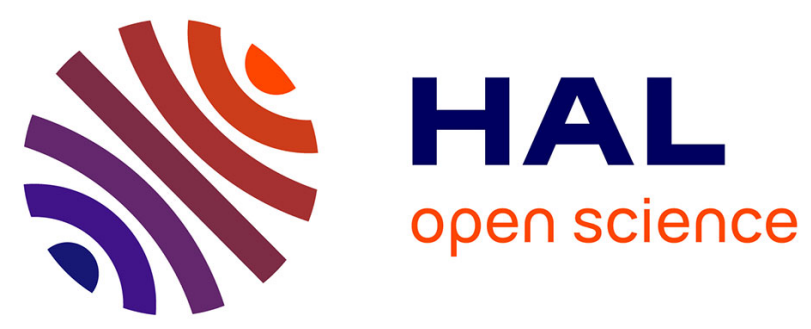

\title{
Finite Eilenberg Machines
}

\author{
Benoit Razet
}

\section{- To cite this version:}

Benoit Razet. Finite Eilenberg Machines. [Research Report] INRIA. 2008. inria-00257354v3

\section{HAL Id: inria-00257354 https://hal.inria.fr/inria-00257354v3}

Submitted on 4 Apr 2008

HAL is a multi-disciplinary open access archive for the deposit and dissemination of scientific research documents, whether they are published or not. The documents may come from teaching and research institutions in France or abroad, or from public or private research centers.
L'archive ouverte pluridisciplinaire HAL, est destinée au dépôt et à la diffusion de documents scientifiques de niveau recherche, publiés ou non, émanant des établissements d'enseignement et de recherche français ou étrangers, des laboratoires publics ou privés. 
INSTITUT NATIONAL DE RECHERCHE EN INFORMATIQUE ET EN AUTOMATIQUE

\title{
Finite Eilenberg Machines
}

\author{
Benoît Razet
}

\section{$N^{\circ} 6486$}

February 2008

Thème SYM

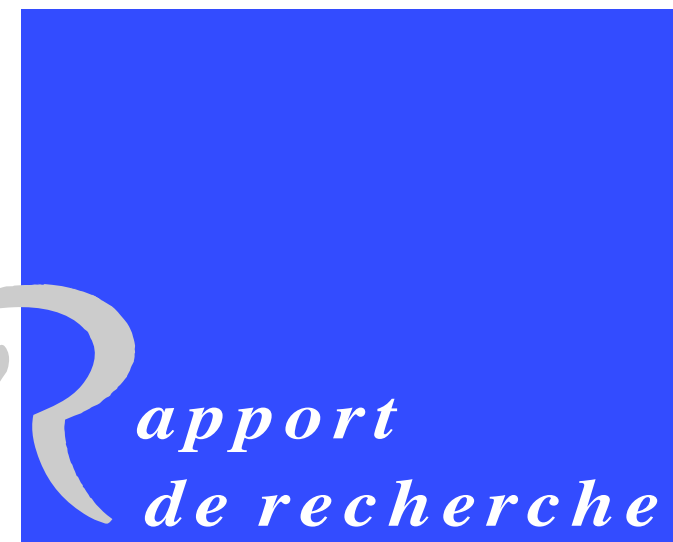





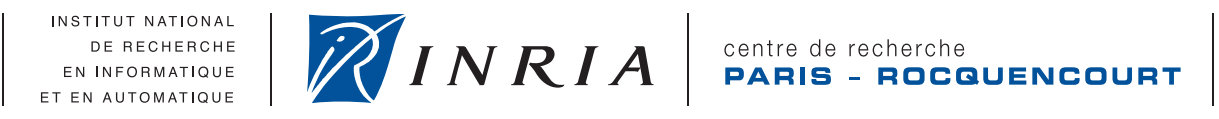

\title{
Finite Eilenberg Machines
}

\author{
Benoît Razet \\ Thème SYM — Systèmes symboliques \\ Équipe-Projet SANSKRIT \\ Rapport de recherche $n^{\circ} 6486$ - February 2008 - 17 pages
}

\begin{abstract}
Eilenberg machines define a general computational model. They are well suited to the simulation of problems specified using finite state formalisms such as formal languages and automata theory. This paper introduces a subclass of them called finite Eilenberg machines. We give a formal description of complete and efficient algorithms which permit the simulation of such machines. We show that our finiteness restriction ensures a correct behavior of the simulation. Interpretations of this restriction are studied for the particular cases of non-deterministic automata (NFA) and rational transducers, leading to applications to computational linguistics. The given implementation provides a generic simulation procedure for any problem encoded as a composition of finite Eilenberg machines.
\end{abstract}

Key-words: Automata, formal languages, Eilenberg machines 


\section{Finite Eilenberg Machines}

Résumé : Les machines d'Eilenberg définissent un modèle de calcul général. Elles permettent de spécifier et résoudre des problèmes de la théorie des langages formels. Ce travail introduit une sous-classe de ces machines qu'on appelle les machines d'Eilenberg finies. De telles machines se simulent à l'aide d'un algorithme effectif pour lequel une spécification formelle est fournie. Nous prouvons que le critère de finitude assure le bon comportement de l'algorithme. Nous discutons aussi de l'impact de ce critère dans le cas où de telles machines sont en fait des automates ou des transducteurs rationnels. Ceci légitime cette étude dans le cadre de la linguistique computationnelle.

Mots-clés : Automates, langages formels, machines d'Eilenberg 


\section{Introduction}

Samuel Eilenberg introduced in the chapter 10 of his book [4], published in 1974, a notion of Machine which he claimed to be a very efficient tool for studying formal languages of the Chomsky hierarchy. They are sometimes referred to as $X$-machines. Many variants have appeared in the last twenty years [1] in several scientific domains different from formal languages.

Eilenberg machines define a general computational model. Assumed given an abstract data set $X$ (it motivates $X$-machine terminology), a machine is defined as an automaton labelled with binary relations on $X$. Two generalizations result from this. Firstly, the set $X$ abstracts the traditional tape used by automata on words, transducers etc. Secondly, compared to functions, binary relations give a built-in notion of non-determinism. Many translations of other machines into Eilenberg machines are given in [4]: automata, transducers, real-time transducers, two-way automata, push-down automata and Turing machines.

The remainder of this paper recalls the definitions of Eilenberg machines in Section 1. It also introduces a new subclass of them called finite Eilenberg machines. The Section 2 motivates their utility discussing two examples. Firstly, we show that any non-deterministic finite automaton (NFA) may be encoded as a finite Eilenberg machine solving its word problem. Secondly, we discuss rational relations and transducers providing encodings into machines solving the three problems of recognition, synthesis and analysis. Resulting machines might not satisfy our finiteness restriction. We discuss the ones for which it is the case considering length-preserving relations and furthermore a variant of positive rational relations. Applications are also motivated by computational linguistics. The next two sections provide algorithms simulating finite Eilenberg machines. Since relations are central to the considered machines, the Section 3 proposes an encoding of them using streams. The Section 4 gives a formal description of algorithms which permit the simulation of finite Eilenberg machines in the spirit of the reactive engine introduced in [5]. Formal proofs are given ensuring the simulation is correct.

\section{Finite Eilenberg machines}

We consider a monoid with carrier $M$, · an associative product on $M$ and 1 its unit element. A finite monoid automaton $\mathcal{A}$ over $M$, also called a $M$-automaton, is a tuple $(Q, \delta, I, T)$ with $Q$ a finite set of elements called states, $\delta$ a function from $Q$ to finite subsets of $(M \times Q)$ called the transition function, $I$ a subset of $Q$ of initial states and $T$ a subset of $Q$ of terminal states.

A path $p$ is a sequence $p=q_{0} \stackrel{m_{1}}{\longrightarrow} q_{1} \stackrel{m_{2}}{\longrightarrow} \cdots \stackrel{m_{n}}{\longrightarrow} q_{n}$ with $n \in \mathbb{N}$ and $\forall i \leq n q_{i} \in$ $Q, \forall i<n m_{i+1} \in M$ and $\forall i<n\left(m_{i+1}, q_{i+1}\right) \in \delta\left(q_{i}\right)$. The path $p$ is successful when $q_{0} \in I$ and $q_{n} \in T$; its length is $n$. The label of $p$ written $\bar{p}$ is 1 if $n=0$ or $m_{1} \cdot \ldots \cdot m_{n}$ otherwise. Finally, the behavior of the $M$-automaton $\mathcal{A}$, written $|\mathcal{A}|$, is defined as the set of all labels of successful paths of $\mathcal{A}$. We introduce the

$\mathrm{RR} \mathrm{n}^{\circ} 6486$ 
type of $\mathcal{A}$, written $\Phi_{\mathcal{A}}$, as the finite subset of $M$ of elements appearing in the image of $\delta$ :

$$
\Phi_{\mathcal{A}}=\left\{m \in M \mid \exists q q^{\prime} \in Q,\left(m, q^{\prime}\right) \in \delta(q)\right\} .
$$

Let us now precise some notations for relations which are central to the remainder of this paper. A relation $\rho$ from some set $\mathcal{D}$ to a set $\mathcal{D}^{\prime}$ written $\rho: \mathcal{D} \rightarrow \mathcal{D}^{\prime}$ is a set of pairs from $\wp\left(\mathcal{D} \times \mathcal{D}^{\prime}\right)$. The functional notation of its type is justified by the isomorphism between $\wp\left(\mathcal{D} \times \mathcal{D}^{\prime}\right)$ and $\mathcal{D} \rightarrow \wp\left(\mathcal{D}^{\prime}\right)$. The converse of a relation $\rho: \mathcal{D} \rightarrow \mathcal{D}^{\prime}$ is written $\rho^{-1}: \mathcal{D}^{\prime} \rightarrow \mathcal{D}$. Let us use $\rho(d)$ as notation for $\left\{d^{\prime} \mid d^{\prime} \in \mathcal{D}^{\prime},\left(d, d^{\prime}\right) \in \rho\right\}$.

Let us recall Eilenberg's definition of machines.

Let $\mathcal{D}$ be an arbitrary set called the data (it replaces the original notation $X$ ). We consider the set $R_{\mathcal{D}}$ of binary relations from $\mathcal{D}$ to $\mathcal{D}$. We consider here the relations monoid $R_{\mathcal{D}}$ with the relation composition $\circ$ as associative product and the identity relation $i d$ as unit element. A $\mathcal{D}$-machine $\mathcal{M}$ is a $R_{\mathcal{D}^{-}}$ automaton $(Q, \delta, I, T)$. With respect to the previous definitions the label of a path $p=q_{0} \stackrel{\phi_{1}}{\longrightarrow} q_{1} \stackrel{\phi_{2}}{\longrightarrow} \cdots \stackrel{\phi_{n}}{\longrightarrow} q_{n}$ is the composition of relations $\bar{p}=\phi_{1} \circ \cdots \circ \phi_{n}$. The behavior of $\mathcal{M}$ as an automaton, $|\mathcal{M}|$, is the set of relations of all labels of successful paths. The distinction between an automaton and a machine lies in the use of the union operation available on relations. The machine $\mathcal{M}$ defines a particular relation, written $\|\mathcal{M}\|$, as the relation union extended over all relations in $|\mathcal{M}|$ :

$$
\|\mathcal{M}\|=\bigcup_{\rho \in|\mathcal{M}|} \rho .
$$

We call the relation $\|\mathcal{M}\|$ the characteristic relation of the machine $\mathcal{M}$. We have given until now what we call the kernel of an Eilenberg machine which refers only to the automaton part.

The complete description of an Eilenberg machine requires what we call its interface. That is, consider $\mathcal{D}_{-}$and $\mathcal{D}_{+}$be two sets called respectively the input and output sets, an input relation $\phi_{-}: \mathcal{D}_{-} \rightarrow \mathcal{D}$ and an output relation $\phi_{+}: \mathcal{D} \rightarrow \mathcal{D}_{+}$. Intuitively, the relation $\phi_{-}$feeds the kernel with inputs and $\phi_{+}$ interprets kernel results as outputs. A machine kernel with its interface defines a relation $\rho: \mathcal{D}_{-} \rightarrow \mathcal{D}_{+}$as

$$
\rho=\phi_{-} \circ\|\mathcal{M}\| \circ \phi_{+} .
$$

The usefulness of kernel and interfaces will be clear with examples provided in section 2 .

Remark 1.1 (on modularity of Eilenberg machines). Any Eilenberg machine $\mathcal{M}$ of type $\Phi_{\mathcal{M}}$ defines a characteristic relation $\|\mathcal{M}\|$ that may belong to a type $\Phi_{\mathcal{M}^{\prime}}$ of another Eilenberg machine $\mathcal{M}^{\prime}$. This gives an idea that Eilenberg machines describe a modular computational model.

We are now going to introduce a new subclass of Eilenberg machines. For this purpose let us first define useful notions specific to machines rather than 
automata. Let us consider a $\mathcal{D}$-machine $\mathcal{M}=(Q, \delta, I, T)$. We call cell a pair $c=(d, q)$ of $\mathcal{D} \times Q$. An edge is a triple $\left((d, q), \phi,\left(d^{\prime}, q^{\prime}\right)\right)$, written $(d, q) \stackrel{\phi}{\longrightarrow}\left(d^{\prime}, q^{\prime}\right)$ and satisfying the two following conditions $\left(\phi, q^{\prime}\right) \in \delta(q)$ and $d^{\prime} \in \phi(d)$. A trace is a sequence of consecutive edges $t=c_{0} \stackrel{\phi_{1}}{\longrightarrow} c_{1} \cdots \stackrel{\phi_{n}}{\longrightarrow} c_{n}$. The integer $n$ is the length of the trace. The cell $c_{0}$ is called its beginning and $c_{n}$ its end. For each data $d$ and state $q$, the cell $(d, q)$ defines a null trace with itself as beginning and end. A cell $(d, q)$ is said to be terminal whenever $q$ is terminal. A trace $t$ is said to be terminal when its end is terminal. Remark that each trace can be projected as the corresponding path when data are forgotten.

\section{Definition 1.1.}

1. Let $\mathcal{D}_{1}$ and $\mathcal{D}_{2}$ be two sets, we say that a relation $\rho: \mathcal{D}_{1} \rightarrow \mathcal{D}_{2}$ is locally finite iff for all data $d$ in $\mathcal{D}_{1}$ the set $\rho(d)$ is finite.

2. We say that a machine $\mathcal{M}$ is locally finite iff every relation $\phi$ in $\Phi_{\mathcal{M}}$ is locally finite.

3. The machine $\mathcal{M}$ is globally finite iff its characteristic relation $\|\mathcal{M}\|$ is locally finite.

4. The machine $\mathcal{M}$ is nœtherian iff there is no infinite trace

$$
c_{0} \stackrel{\phi_{1}}{\longrightarrow} c_{1} \cdots \stackrel{\phi_{n}}{\longrightarrow} c_{n} \cdots .
$$

5. The machine $\mathcal{M}$ is called finite iff it is locally finite and notherian.

Remark 1.2. A locally finite machine may or may not be globally finite and conversely a globally finite machine may or may not be locally finite.

Proposition 1.1. If the machine $\mathcal{M}$ is finite then it is globally finite.

Proof. Using König's lemma; the locally finite condition corresponds to the finite branching condition and the notherian condition to the non existence of infinite traces.

Corollary 1.1. Let $\phi_{-}$and $\phi_{+}$be two partial functions. If the machine $\mathcal{M}$ is finite with interface $\phi_{-}$and $\phi_{+}$then the relation $\phi_{-} \circ\|\mathcal{M}\| \circ \phi_{+}$is locally finite.

We are interested in such finite Eilenberg machines because we show in section 4 that their simulation may be implemented in a complete fashion taking advantage of the finiteness and nœtherian properties. Before that, we discuss examples and applications of finite Eilenberg machines.

$\mathrm{RR} \mathrm{n}^{\circ} 6486$ 


\section{Examples and applications}

Examples from Eilenberg showing his machine model implements many others used "relabelings" which are formally presented in [10]:

Definition 2.1. Let $M_{1}$ and $M_{2}$ be two monoids, $\alpha: M_{1} \rightarrow M_{2}$ a monoid morphism and $\mathcal{A}_{1}=\left(Q, \delta_{1}, I, T\right)$ a $M_{1}$-automaton. A $M_{2}$-automaton $\mathcal{A}_{2}=$ $\left(Q, \delta_{2}, I, T\right)$ is a relabelling of $\mathcal{A}_{1}$ by $\alpha$ iff $\mathcal{A}_{2}$ is a copy of $\mathcal{A}_{1}$ except that each label $m$ in the image of $\delta_{1}$ is replaced by $\alpha(m)$ in $\delta_{2}$. That is $\Phi_{\mathcal{A}_{2}}=\alpha\left(\Phi_{\mathcal{A}_{1}}\right)$, the morphism $\alpha$ being extended to sets.

The main result on relabellings is the following:

Lemma 2.1. Let $\alpha: M_{1} \rightarrow M_{2}$ be a monoid morphism, $\mathcal{A}_{1}$ a $M_{1}$-automaton and $\mathcal{A}_{2}$ a $M_{2}$-automaton relabelled from $\mathcal{A}_{1}$ by $\alpha$. Then the behavior of $\mathcal{A}_{2}$ is the image by $\alpha$ of the behavior of $\mathcal{A}_{1}$, i.e.:

$$
\left|\mathcal{A}_{2}\right|=\alpha\left(\left|\mathcal{A}_{1}\right|\right)
$$

Proof. Classic e.g. [10].

Eilenberg provided relabellings that translate well-known finite state formalisms into machines. We are going to discuss cases when the resulting machines are in fact finite Eilenberg machines. For this purpose let us recall some notions. We consider a finite set $\Sigma$ of letters called the alphabet. We consider the free monoid $\Sigma^{*}$ of words over $\Sigma$ with the word concatenation as monoid product and the empty word $\epsilon$ as unit element. Formal languages are sets of words. Four basic operations on words are to be considered for defining Eilenberg machines for the next examples. For each letter $\sigma$ of $\Sigma$ :

- $L_{\sigma}=\left\{(w, \sigma w) \mid w \in \Sigma^{*}\right\}$

- $R_{\sigma}=\left\{(w, w \sigma) \mid w \in \Sigma^{*}\right\}$

- $L_{\sigma}^{-1}=\left\{(\sigma w, w) \mid w \in \Sigma^{*}\right\}$

- $R_{\sigma}^{-1}=\left\{(w \sigma, w) \mid w \in \Sigma^{*}\right\}$

The $L$ and $R$ denotations indicate operations respectively on the left or the right of a word. The last two relations are respectively the converse relations of the first ones. We will also use the identity relation written $i d_{\Sigma^{*}}$ as the relation $\left\{(w, w) \mid w \in \Sigma^{*}\right\}$.

Remark 2.1. Relations $L_{\sigma}, R_{\sigma}, L_{\sigma}^{-1}$ and $R_{\sigma}^{-1}$ described above are in fact partial functions, thus they are locally finite relations.

\subsection{NFA and finite Eilenberg machines}

We consider here an alphabet $\Sigma$ and words as elements of $\Sigma^{*}$. A NFA on alphabet $\Sigma$ is a $\Sigma^{*}$-automaton $\mathcal{A}$ such that $\Phi_{\mathcal{A}} \subseteq \Sigma(\epsilon$-transitions are not 
allowed). The set of words $|\mathcal{A}|$, the behavior of $\mathcal{A}$, is a formal language that belongs to the class of rational languages.

Let us define a relabelling procedure translating any NFA into an Eilenberg machine solving its word problem. Let $\mathcal{A}=(Q, \delta, I, T)$ be a NFA. We choose a data set $\mathcal{D}=\Sigma^{*}$. Since $\Phi_{\mathcal{A}} \subseteq \Sigma$, we define our morphism $\alpha$ on each element of $\Sigma$ as $\alpha(\sigma)=L_{\sigma}^{-1}$. Thus the machine $\mathcal{M}$ relabelled from $\mathcal{A}$ by $\alpha$ has the following characteristic relation: $\|\mathcal{M}\|=\left\{\left(w w^{\prime}, w^{\prime}\right)|w \in| \mathcal{A} \mid, w^{\prime} \in \Sigma^{*}\right\}$. That is, a given input $w \cdot w^{\prime}$ is truncated by the word $w$ recognized by the automaton $\mathcal{A}$. The machine $\mathcal{M}$ is a finite Eilenberg machine because it satisfies the locally finite condition due to remark 2.1 and the nœtherian condition because for every edge $(w, q) \stackrel{L_{\sigma}^{-1}}{\longrightarrow}\left(w^{\prime}, q^{\prime}\right)$ we have $\left|w^{\prime}\right|=|w|-1$, this shows that the length of traces is bounded by the length of their beginning word in their initial cell and thus there may not be infinite traces.

The encoding is complete when considered this finite Eilenberg machine with the following interface: $\mathcal{D}_{-}=\Sigma^{*}$, the input relation $\phi_{-}=i d_{\Sigma^{*}}, \mathcal{D}_{+}=\mathbb{B}$ the Boolean set composed of the two values $T$ and $\perp$ and the output function $\phi_{+}: \Sigma^{*} \rightarrow \mathbb{B}$ defined by $\phi_{+}(w)$ being $\top$ when $w=\epsilon$ and $\perp$ otherwise. Now we have $\left(\phi_{-} \circ\|\mathcal{M}\| \circ \phi_{+}\right)^{-1}(\top)=|\mathcal{A}|$. It shows that the relabelling is correct.

Remark 2.2. Languages recognized by NFA with $\epsilon$-transitions may also be embedded in such Eilenberg machines but they would not necessarily satisfy the notherian condition since there may be cycles of $\epsilon$-transitions. Conversely a NFA with no such cycle is a finite Eilenberg machine.

\subsection{Rational transducers and finite Eilenberg machines}

Let $\Sigma$ and $\Gamma$ be two finite alphabets. The empty word $\epsilon$ will denote both empty words for $\Sigma^{*}$ and for $\Gamma^{*}$. We consider here the monoid $\Sigma^{*} \times \Gamma^{*}$ with its traditional concatenation as associative product and the pair $(\epsilon, \epsilon)$ as unit element. A rational transducer from $\Sigma^{*}$ to $\Gamma^{*}$ is a monoid automaton $\mathcal{A}$ over $\Sigma^{*} \times \Gamma^{*}$ such that its type $\Phi_{\mathcal{A}} \subseteq(\Sigma \times \epsilon) \cup(\epsilon \times \Gamma)$. The subset of pairs of words from $|\mathcal{A}|$, the behavior of $\mathcal{A}$, defines a relation which belongs to the subclass of rational relations. A transducer defines a relation which solves the three following problems:

1. Recognition Given a couple of words $\left(w, w^{\prime}\right)$ of $\Sigma^{*} \times \Gamma^{*}$, does $\left(w, w^{\prime}\right)$ belong to $|\mathcal{A}|$.

2. Synthesis Given a word $w$ in $\Sigma^{*}$ compute the set $|\mathcal{A}|(w)$ of words from $\Gamma^{*}$.

3. Analysis Given a word $w$ in $\Gamma^{*}$ compute the set $|\mathcal{A}|^{-1}(w)$ of words from $\Sigma^{*}$.

Let us now provide the relabelling of the transducer $\mathcal{A}=(Q, \delta, I, T)$ for solving the recognition problem. The morphism $\alpha$ is completely defined on elements of $\Phi_{\mathcal{A}}$ with $\alpha(\sigma, \epsilon)=L_{\sigma}^{-1} \times i d_{\Gamma^{*}}$ and $\alpha(\epsilon, \gamma)=i d_{\Sigma^{*}} \times L_{\gamma}^{-1}$. The machine $\mathcal{M}=\alpha(\mathcal{A})$ is nœtherian since each transition of $\mathcal{M}$ consumes either

$\mathrm{RR} \mathrm{n}^{\circ} 6486$ 
a letter from a word of $\Sigma^{*}$ or a word of $\Gamma^{*}$. We complete the definition of the machine with the set $\mathcal{D}_{-}=\Sigma^{*} \times \Gamma^{*}$, the input relation $\phi_{-}=i d_{\Sigma^{*} \times \Gamma^{*}}$, the set $\mathcal{D}_{+}=\mathbb{B}$ and output relation $\phi_{+}$with $\phi_{+}(\epsilon, \epsilon)=\top$ or $\perp$ otherwise. Thus we have

$$
\left(\phi_{-} \circ\|\mathcal{M}\| \circ \phi_{+}\right)^{-1}(\top)=|\mathcal{A}| .
$$

This result concerning the recognition problem for rational relations is similar to the one concerning the word problem of NFA.

We now consider the relabelling for the synthesis problem. The morphism $\alpha$ is defined on $\Phi_{\mathcal{A}}$ such that $\alpha(\sigma, \epsilon)=L_{\sigma}^{-1} \times i d_{\Gamma^{*}}$ and $\alpha(\epsilon, \gamma)=i d_{\Sigma^{*}} \times R_{\gamma}$.

This encoding is complete when we consider the Eilenberg machine $\mathcal{M}=\alpha(\mathcal{A})$ with the following interface: $\mathcal{D}_{-}=\Sigma^{*}, \mathcal{D}_{+}=\Gamma^{*}$, the input relation $\phi_{-}=$ $\left\{(w,(w, \epsilon)) \mid w \in \Sigma^{*}\right\}$ and the output relation $\phi_{+}=\left\{\left(\left(\epsilon, w^{\prime}\right), w^{\prime}\right) \mid w^{\prime} \in \Gamma^{*}\right\}$. Then the relation described by this machine is:

$$
\left(\phi_{-} \circ\|\mathcal{M}\| \circ \phi_{+}\right)=|\mathcal{A}| .
$$

Such an Eilenberg machine is trivially proved to be locally finite. But in this case the Eilenberg machine $\alpha(\mathcal{A})$ might not always be nœtherian: for example, a cycle in the machine labelled only by relations of the form $i d_{\Sigma^{*}} \times R_{\gamma}$ generates possibly infinite traces.

Nevertheless such configurations could not happen for length-preserving relations implemented by resynchronized transducers of type $\Sigma \times \Gamma$ which are compiled into finite Eilenberg machines. We have furthermore:

Proposition 2.1. For any rational relation implemented by a trimmed rational transducer $\mathcal{A}$ of type $\Phi_{\mathcal{A}} \subseteq(\Sigma \times \epsilon) \cup(\epsilon \times \Gamma)$, the following conditions are equivalent:

1. For all $w \in \Sigma^{*}$ the set $|\mathcal{A}|(w)$ is finite.

2. There is no cycle of type $(\epsilon \times \Gamma)$ in $\mathcal{A}$.

3. The machine $\mathcal{M}=\alpha(\mathcal{A})$ is notherian.

Proof.

$(1) \Rightarrow(2)$ By contraposition. Assume that there exists such a cycle at some state $q$. The transducer being trimmed the state $q$ is accessible and coaccessible. Thus there exists a word $w_{1}$ of $\Sigma^{*}$ defining a path leading to $q$ from an initial state and there exists a word $w_{2}$ of $\Sigma^{*}$ defining a path leaving from $q$ and arriving on a terminal state. We conclude that $|\mathcal{A}|\left(w_{1} w_{2}\right)$ is infinite. Thus (1) is false.

$(2) \Rightarrow(3)$ Assume the proposition (2) true. Since $\mathcal{A}$ is a finite automaton there exists an integer $n$ bounding paths with labels of type $(\epsilon \times \Gamma)$. Let $w$ be a word of $\Sigma^{*}$ and let $i$ be the length of $w$ then the length of any trace beginning with $\left(w, w^{\prime}\right)$ for any $w^{\prime} \in \Gamma^{*}$ is at most $n \times i$. Thus $\mathcal{M}$ is nœtherian. 
$(3) \Rightarrow$ (1) From corollary 1.1.

The proposition 2.1 shows that for the particular case of rational transducers, the implication of corollary 1.1 becomes an equivalence. This result is to be related to positive rational relations for which Eilenberg gave a resynchronization procedure.

The analysis problem might be solved similarly to the synthesis using $R_{\sigma}$ instead of $L_{\sigma}^{-1}$ and $L_{\gamma}^{-1}$ instead of $R_{\gamma}$.

\subsection{Applications to computational linguistics}

Automata, transducers and more generally finite state machines are a popular technology for solving many computational linguistics problems [9]. We believe that Eilenberg machines are an efficient tool for this purpose. In fact, our restriction of finite Eilenberg machines is the formalism underlying the works concerning general morphological and phonetical modelizations [5,6] which have been applied to the Sanskrit language modelization. Furthermore this application needs the modularity of such Eilenberg machine as sketched in the remark 1.1. In the following we provide algorithms that simulate in a complete fashion any finite Eilenberg machine. They are given with a complete formalization.

\section{Streams representing relations}

We consider now that the data set $\mathcal{D}$ is representable as an abstract ML datatype. We recall that unit is the singleton ML datatype containing the unique value denoted (). In our implementation we will use streams which are objects for enumerating on demand. In ML notation stream values are encoded with the following type parametrized with $\mathcal{D}$ :

$$
\begin{aligned}
& \text { type stream } \mathcal{D}=\mid E O S \\
& \text { Stream of } \mathcal{D} \times(\text { delay } \mathcal{D}) \\
& \text { and delay } \mathcal{D}=\text { unit } \rightarrow \text { stream } \mathcal{D} \text {; }
\end{aligned}
$$

A stream value is either the empty stream EOS ("End of Stream") for encoding the empty enumeration or else a value Stream $d$ del that provides the new element $d$ of the enumeration and a value del as a delayed computation of the rest of the enumeration. Since ML computes with the restriction of $\lambda$-calculus to weak reduction, a value of type delay $\mathcal{D}$ such as del is delayed because it is a functional value. This well known technique permits computation on demand. Note that this technique would not apply in a programming language evaluating inside a function body (strong reduction in $\lambda$-calculus terminology).

ML being a Turing-complete programming language, not all ML functions terminate and since our streams contain function values we shall restrict their computational power:

\section{Definition 3.1.}

1. The $M L$ function $f:$ unit $\rightarrow \alpha$ is said to be total iff the evaluation of $f()$ terminates (yielding a value of type $\alpha$ ).

$\mathrm{RR} \mathrm{n}^{\circ} 6486$ 
2. The $M L$ stream str: stream $\mathcal{D}$ is said to be progressive iff

- either str is EOS

- or else str is of the form Stream d $\mathrm{f}$ with $\mathrm{f}$ total, and $\mathrm{f}$ () is progressive.

We define the head function $h d$ from non-empty streams to data defined as follows: $h d($ Stream $d d e l)=d$. We define also the tail function $t l$ from streams to streams as $t l(E O S)=E O S$ and $t l($ Stream $d$ del $)=\operatorname{del}()$. Let $n$ be an integer, we introduce the function $t l^{n}$ that iterates the $t l$ function $n$ times:

$$
\begin{cases}t l^{0}(s t r) & =s t r \\ t l^{n+1}(s t r) & =t l^{n}(t l(s t r))\end{cases}
$$

We introduce the predicate $\operatorname{InStream}(d, s t r)$ that checks whether a data $d$ appears in the stream str:

Definition 3.2. InStream $(d, s t r)$ is true iff there exists an integer $n$ such that $\left(t l^{n}(s t r)\right)$ is non-empty and $h d\left(t l^{n}(s t r)\right)=d$.

Definition 3.3. A progressive stream str is finite iff there exists an integer $n$ such that $t l^{n}(s t r)=E O S$.

The length of a finite stream str, written $|s t r|$, is defined inductively as follows:

$$
\begin{cases}\mid \text { EOS } \mid & =0 \\ \mid \text { Stream d del } \mid & =1+|\operatorname{del}()|\end{cases}
$$

All finite streams of positive length end with a value of type delay $\mathcal{D}$ that associates to the unit element () the EOS stream announcing the end of the enumeration, typically:

value delay_eos $=$ fun ()$\rightarrow E O S ;$

We consider now relations of $R_{\mathcal{D}}$ representable as ML functions of the following type:

type relation $\mathcal{D}=\mathcal{D} \rightarrow$ stream $\mathcal{D}$

That is, if a relation rel of type relation $\mathcal{D}$ corresponds to a relation $\rho$ of $R_{\mathcal{D}}$ then:

$$
\forall d d^{\prime} \in \mathcal{D}, d^{\prime} \in \rho(d) \Leftrightarrow \operatorname{InStream}\left(d^{\prime} \text {, rel } d\right)
$$

In the following we will use this technique only for representing locally finite relations. That is, relations are encoded using finite streams which are progressive by definition 3.3. From now on, we shall assume that our Eilenberg machines are effective in the sense that their data domain $\mathcal{D}$ are implemented as an $\mathrm{ML}$ datatype and that every relation used in their labeling is progressive. 


\section{A reactive engine for finite Eilenberg machines}

We provide an implementation for the simulation of finite Eilenberg machines using higher-order recursive definitions. Algorithms are presented using ML notations which are directly executable in the OCaml programming language [7]. An essential feature of our formal notations is to possibly compose parametrized modules called functors. Algorithms are variants of the reactive engine [5]. They are presented completely using only a dozen of elegant definitions.

\subsection{The reactive engine}

Let $\mathcal{M}=(Q, \delta, I, T)$ be a $\mathcal{D}$-machine. We specify $\mathcal{M}$ as a module with the following signature:

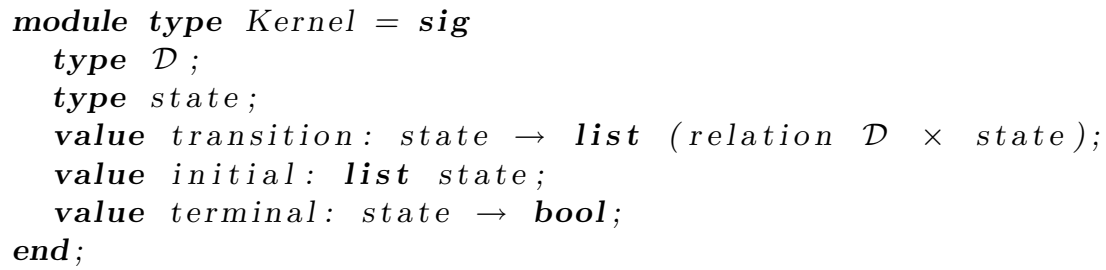

The type parameter state encodes the set $Q$, the function transition encodes the function $\delta$, the value initial encodes the initial states $I$ as a list and the function terminal encodes terminal states $T$ seen as a predicate.

We aim at providing the algorithms implementing the characteristic relation of $\mathcal{M}$. For this purpose we use a functor that is a module parametrized by a Kernel machine. We call Engine this functor declared as the following:

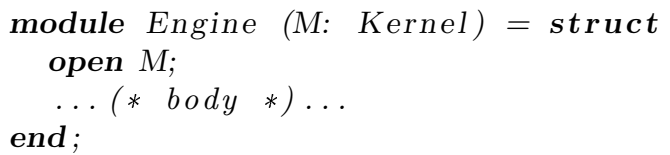

Firstly, the body of the functor contains type declarations:

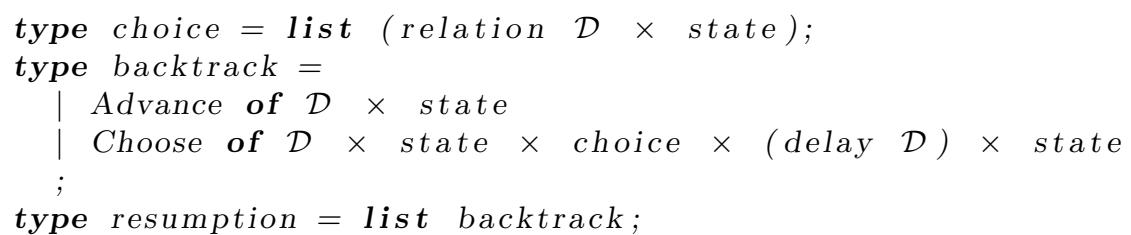

The type choice is an abbreviation for the list of transitions of the machine as used in the machine $M$. Eilenberg machines are possibly non-deterministic and need thus a backtracking mechanism for their implementation. Values of type backtrack allow to save the multiple choices due to the non-deterministic nature of the machine. The enumerating procedure will stack such backtrack values in a resumption of type resumption.

Secondly, the engine contains four functions. Three of them are internal and mutually recursive: react, choose and continue. They perform the nondeterministic search enriching the resumption as the computation goes on. The

$\mathrm{RR} \mathrm{n}^{\circ} 6486$ 
computation is performed in a depth-first search manner stacking the transition choices and streams within backtrack values in the resumption:

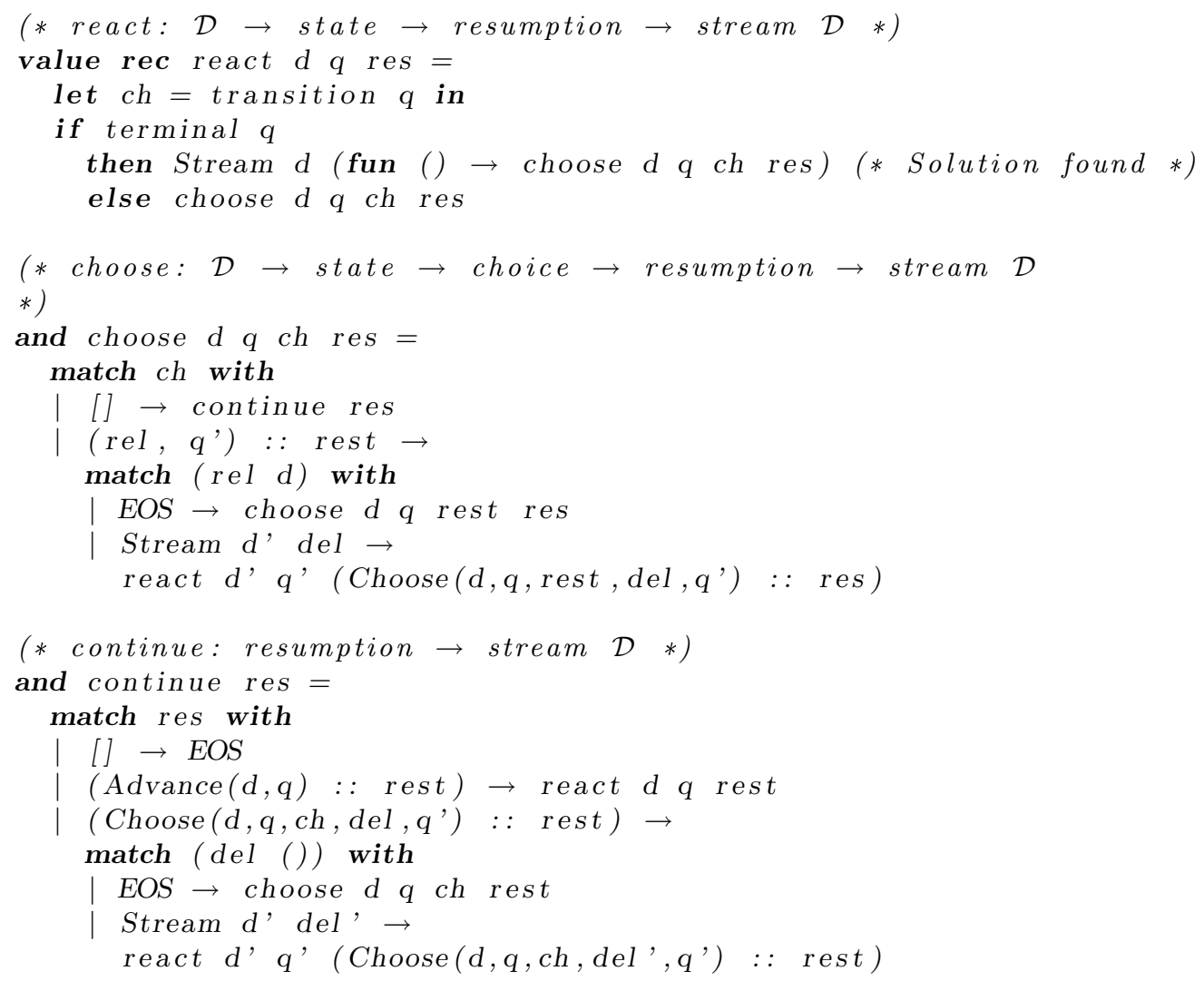

The function react checks whether the state is terminal and then provides an element of the stream delaying the rest of the exploration calling to the function choose. This function choose performs the non-deterministic search over transitions, choosing them in the natural order induced by the list data structure. The function continue manages the backtracking mechanism and the enumeration of finite streams of relations, it always chooses to backtrack on the last pushed value in the resumption. Remark that these three mutually recursive functions do not use any side effect and are written in a pure functional style completely tail-recursive using the resumption as a continuation mechanism.

The machine $\mathcal{M}$ implemented as a module $M$ has its characteristic relation $\|\mathcal{M}\|$ simulated by the following function:

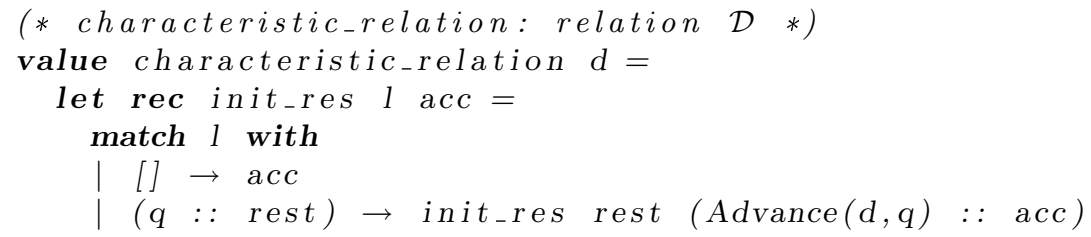


in continue (init_res initial []);

The function characteristic_relation first initializes the resumption with Advance backtrack values for each initial state and then call the function continue on it. We summarize the presented algorithms as follows: the machine $\mathcal{M}$ implemented as a module $M$ has its characteristic relation $\|\mathcal{M}\|$ simulated by Engine(M). characteristic_relation, the function given by the instantiation of functor Engine with module $M$. We now provide the formalization with all arguments ensuring the correctness of our so-called reactive engine.

\subsection{Formalization}

The formalization is inspired by the original one for the reactive engine [5]. It uses the multiset ordering technique as presented by Dershowitz and Manna [3] to prove the termination of our algorithms. It also gives us a useful nœetherian induction principle for the soundness and completeness proofs.

We formalize the fact that data $d$ and $d^{\prime}$ are in relation by the characteristic relation of $\mathcal{M}$ using the predicate Solution $\left(d, d^{\prime}\right)$ which is true iff there exists an initial state $q$ and a terminal trace $t$ beginning with cell $(d, q)$ and ending with data $d^{\prime}$.

Theorem 4.1. If the machine $\mathcal{M}$ is finite then for all data $d$ and $d^{\prime}$,

$$
\operatorname{InStream}\left(d^{\prime}, \text { characteristic_relation } d\right) \Leftrightarrow \operatorname{Solution}\left(d, d^{\prime}\right) \text {. }
$$

One direction of the equivalence of the theorem corresponds to the soundness of the algorithm and the other to its completeness.

Let us define WellFormedBack(b), an invariant on backtrack values $b$ met during the computation of the three mutually recursive functions inside an execution of the function characteristic_relation :

1. WellFormedBack $(\operatorname{Advance}(d, q))$ for every data $d$ and state $q$.

2. WellFormedBack(Choose $\left.\left(d, q, \operatorname{ch}, \operatorname{del}, q^{\prime}\right)\right)$ when there exists a relation rel such that the following conditions are verified:

- $\left(\right.$ rel,$\left.q^{\prime}\right) \in($ transition $q)$,

- $\forall d^{\prime}, \operatorname{InStream}\left(d^{\prime}\right.$, del ()$) \Rightarrow \operatorname{InStream}\left(d^{\prime},(\right.$ rel d $\left.)\right)$,

- $\forall e, e \in c h \Rightarrow e \in($ transition $q)$.

We extend the property WellFormedBack of backtrack values to resumptions by:

$$
\text { WellFormedRes(res })=(\forall b, b \in \text { res } \Rightarrow \text { WellFormedBack }(b)) \text {. }
$$

Before proving the theorem 4.1 we prove the termination of the algorithms. The machine $\mathcal{M}$ being assumed nœtherian, cells are partially-ordered with the edge relation and this ordering is notherian. Consider triples of the form $\left\langle c, n_{1}, n_{2}\right\rangle$ with $c$ being a cell and the other parameters $n_{1}$ and $n_{2}$ integers. Such triples together with extension of lexical ordering (using the traditional ordering on

RR n 6486 
natural numbers) form also a nœtherian partial-ordering. In the following we shall use the extension on multisets of such triples and its multiset-ordering [3].

Now we shall define a function $\chi$ which associates a multiset of triples to each function call of react, choose and continue. Proving that the multiset decreases along recursive calls shows that the functions terminate.

Definition 4.1. If res is a resumption we define $\chi($ res $)$ as the multiset of all $\chi($ back $)$, for back a backtrack value in res, where

$$
\begin{gathered}
\chi\left(\text { Choose }\left(d, q, \text { ch }, \operatorname{del}, q^{\prime}\right)\right)=\langle(d, q),|\operatorname{ch}|,|\operatorname{del}()|+1\rangle \\
\chi(\operatorname{Advance}(d, q))=\langle(d, q), \kappa(q)+1,0\rangle
\end{gathered}
$$

with $\kappa(q)=\mid$ transition $q \mid+1$. We now associate such multisets to every function invocation react, choose and continue:

$$
\begin{gathered}
\chi(\text { react d q res })=\{\langle(d, q), \kappa(q), 0\rangle\} \oplus \chi(\text { res }) \\
\chi(\text { choose } d q \text { ch res })=\{\langle(d, q),| \text { ch }|, 0\rangle\} \oplus \chi(\text { res }) \\
\chi(\text { continue res })=\chi(\text { res })
\end{gathered}
$$

with $\oplus$ being the multiset union.

Using the function $\chi$ we have the following proposition:

Proposition 4.1. If the machine $\mathcal{M}$ is finite, for all res value of type resumption, if WellFormedRes(res) then continue res returns a stream value which is either EOS or (Stream d' del) for some data d' and delay del such that $\chi($ res $) \gg$ $\chi(\operatorname{del}())$.

Proof. We first appropriately strenghten this proposition for functions react and choose. The proof is by simultaneous nœtherian induction over the multiset ordering computed by $\chi$. Inspecting all the branches of the mutually recursive functions react, choose and continue, it is easy to see that $\chi$ decreases.

Corollary 4.1 (Termination). If the machine $\mathcal{M}$ is finite, for all data $d$ the execution of function characteristic_relation applied to d returns a finite (progressive) stream.

Now we consider the predicate $\operatorname{PartSol}\left(c, d^{\prime}\right)$ true iff there exists a terminal trace $t$ beginning with cell $c$ and ending with data $d^{\prime}$.

We extend this predicate on choice values as a PartSolChoice predicate defined as the following: PartSolChoice $\left(d, c h, d^{\prime}\right)$ is true if and only if there exists a relation rel and a state $q_{1}$ with $\left(r e l, q_{1}\right)$ in ch and such that there exists a data $d_{1}$ in the stream rel $d$ and such that PartSol $\left(\left(d_{1}, q_{1}\right), q^{\prime}\right)$ is true.

We define the same kind of predicate for backtrack values called PartSolBack:

1. PartSolBack $\left(\right.$ Advance $\left.(d, q), d^{\prime}\right)$ iff there exists a terminal trace beginning with $(d, q)$ and ending with data $d^{\prime}$. 
2. PartSolBack(Choose $\left(d, q\right.$, ch, del, $\left.q_{1}\right)$, d') iff PartSolChoice $\left(d, c h, d^{\prime}\right)$ is true or else there exists a data $d_{1}$ in the stream del () with $\operatorname{PartSol}\left(\left(d_{1}, q_{1}\right), d^{\prime}\right)$ being true.

We extend the property PartSolBack of backtrack values to resumptions by:

$$
\operatorname{PartSolRes}\left(\text { res, } d^{\prime}\right)=\left(\exists b, b \in \operatorname{res} \wedge \operatorname{PartSolBack}\left(b, d^{\prime}\right)\right) .
$$

Now we may formulate the three following soundness and completeness lemmas for functions react, choose and continue:

Lemma 4.1. If the machine $\mathcal{M}$ is finite then the three following properties are verified:

1. $\forall d q$ res $d^{\prime}$, WellFormedRes $($ res $) \Rightarrow$ $\operatorname{InStream}\left(d^{\prime}\right.$, react $d q$ res $) \Leftrightarrow \operatorname{PartSol}\left((d, q), d^{\prime}\right) \vee \operatorname{PartSolRes}\left(\right.$ res,$\left.d^{\prime}\right)$,

2. $\forall d q$ ch res $d^{\prime}$, WellFormedRes(res $) \Rightarrow$ ch $\subseteq($ transition $q) \Rightarrow$ In Stream $\left(d^{\prime}\right.$, choose $d$ ch res $) \Leftrightarrow$ PartSolChoice $\left(d\right.$, ch, $\left.d^{\prime}\right) \vee$ PartSolRes(res, $\left.d^{\prime}\right)$,

3. $\forall$ res $d^{\prime}$, WellFormedRes(res) $\Rightarrow$ InStream $\left(d^{\prime}\right.$, continue res $) \Leftrightarrow$ PartSolRes(res, $\left.d^{\prime}\right)$.

Proof. The proof is a routine case analysis by simultaneous notherian induction over $\chi$.

Proof of Theorem 4.1. Let $d$ be a data, the execution of characteristic_relation applied to $d$ leads to the execution of continue applied to a resumption value res $=$ (init_res initial []). This resumption res is proved to be composed of backtrack values Advance $(d, q)$ with state $q$ being necessarily an initial state. Also for such a resumption res we have WellFormedRes(res). Applying the third case of the lemma 4.1 we obtain

$$
\operatorname{InStream}\left(d^{\prime}, \text { continue res }\right) \Leftrightarrow \operatorname{PartSolRes}\left(\text { res }, d^{\prime}\right) \text {. }
$$

Also with the considered resumption res, for any data $d^{\prime}$ we have

$$
\text { PartSolRes }\left(\text { res }, d^{\prime}\right) \Leftrightarrow \text { Solution }\left(d, d^{\prime}\right)
$$

because states inside the resumption are initial. Then we conclude by transitivity of the two equivalences.

Termination, soundness and completeness ensure that the function characteristic_relation implements a locally finite relation as a relation $\mathcal{D}$. It illustrates the proposition 1.1 and ensures the correctness of the reactive engine that simulates any finite Eilenberg machine.

$\mathrm{RR} \mathrm{n}^{\circ} 6486$ 


\section{Conclusion}

Eilenberg machines provide a powerful and elegant framework for simulating specifications presented as finite automata variants. Eilenberg gave easy encodings into machines of formalisms at various levels of the Chomsky hierarchy. We have shown that our subclass of finite Eilenberg machines is large enough to support NFA and many transducers. Other examples might be given since our restriction keeps most of the generality brought by original Eilenberg machines. Our machines are not restricted to treatments for the rational level of the Chomsky hierarchy. This particular point makes us believe that finite Eilenberg machines have applications to computational linguistics. In fact they are already efficient for explaining recognition or transduction problems that manipulate two levels of finite state formalisms such as explained in [6] for the modelization of the Sanskrit language. This multi-level ability is a feature of Eilenberg machines that we call modularity. For this purpose implementations need to be lazy. We anticipate future works in this spirit providing lazy algorithms. Our small but efficient reactive engine computes lazily the simulation of any finite Eilenberg machines. Our methodology using higher-order recursive definitions of functional programming language leads to formal proofs amenable to a complete formalization using higher-order logic. Such a formal development is available in the companion paper [8] using the proof assistant for Coq [2].

\section{Acknowledgments}

Gérard Huet and Jean-Baptiste Tristan took an essential part in the elaboration of this paper; I thank them for their intensive participation.

\section{References}

[1] Theory of X-machines. http://www.x-machines.com.

[2] The Coq proof assistant. Software and documentation available on the Web, http://coq.inria.fr/, 1995-2007.

[3] Nachum Dershowitz and Zohar Manna. Proving termination with multiset orderings. Commun. ACM, 22(8):465-476, 1979.

[4] Samuel Eilenberg. Automata, Languages and Machines, volume A. Academic Press, 1974.

[5] Gérard Huet. A functional toolkit for morphological and phonological processing, application to a Sanskrit tagger. J. Functional programming, 15, 2005 .

[6] Gérard Huet and Benoît Razet. The reactive engine for modular transducers. In Jean-Pierre Jouannaud Kokichi Futatsugi and José Meseguer, 
editors, Algebra, Meaning and Computation, Essays Dedicated to Joseph A. Goguen on the Occasion of His 65th Birthday, pages 355-374. SpringerVerlag LNCS vol. 4060, 2006.

[7] Xavier Leroy, Damien Doligez, Jacques Garrigue, and Jérôme Vouillon. The Objective Caml system. Software and documentation available on the Web, http://caml.inria.fr/, 1996-2006.

[8] Benoît Razet. Simulating Eilenberg machines with a reactive engine: Formal specification, proof and program extraction. Research Report 6487, INRIA, 022008.

[9] Emmanuel Roche and Yves Schabes. Finite-state language processing. MIT press, 1997.

[10] Jacques Sakarovitch. Eléments de théorie des automates. Vuibert, Paris, 2003.

$\mathrm{RR} \mathrm{n}^{\circ} 6486$ 


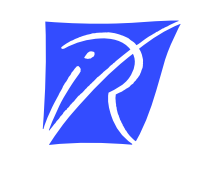

Centre de recherche INRIA Paris - Rocquencourt

Domaine de Voluceau - Rocquencourt - BP 105 - 78153 Le Chesnay Cedex (France)

Centre de recherche INRIA Bordeaux - Sud Ouest : Domaine Universitaire - 351, cours de la Libération - 33405 Talence Cedex

Centre de recherche INRIA Grenoble - Rhône-Alpes : 655, avenue de l'Europe - 38334 Montbonnot Saint-Ismier

Centre de recherche INRIA Lille - Nord Europe : Parc Scientifique de la Haute Borne - 40, avenue Halley - 59650 Villeneuve d'Ascq

Centre de recherche INRIA Nancy - Grand Est : LORIA, Technopôle de Nancy-Brabois - Campus scientifique 615, rue du Jardin Botanique - BP 101 - 54602 Villers-lès-Nancy Cedex

Centre de recherche INRIA Rennes - Bretagne Atlantique : IRISA, Campus universitaire de Beaulieu - 35042 Rennes Cedex

Centre de recherche INRIA Saclay - Île-de-France : Parc Orsay Université - ZAC des Vignes : 4, rue Jacques Monod - 91893 Orsay Cedex

Centre de recherche INRIA Sophia Antipolis - Méditerranée : 2004, route des Lucioles - BP 93 - 06902 Sophia Antipolis Cedex 